\title{
Usando Objetos Educacionais baseados em Realidade Virtual em ambientes de apoio a construção de conhecimento e aprendizagem de técnicas videocirúrgicas.
}

\author{
Luis de França Ferreira*
}

Resumo: Com a popularização das chamadas cirurgias minimamente invasivas, ou videocirurgias como também são conhecidas, os cirurgiões precisam aprender e adquirir habilidades para executar procedimentos radicalmente diferentes daqueles existentes nas cirurgias tradicionais. Os ambientes de aprendizado hoje existentes não dão mais conta da tarefa. São necessárias então, novas alternativas. A Realidade Virtual é uma promissora opção como um artefato de apoio ao aprendizado dessas novas técnicas cirúrgicas. Além disto, é necessário também que estes ambientes sejam ser difundidos na comunidade. $O$ uso de objetos educacionais, baseados em Realidade Virtual e no padrão SCORM ${ }^{T M}$ se apresenta como uma interessante alternativa para a difusão de ambientes de apoio a construção de conhecimento e aprendizagem de técnicas videocirúrgicas altamente qualificados.

Palavras Chaves: Objetos educacionais. Conhecimento. Artefatos digitais. Realidade Virtual. Educação médica. Cirurgias minimamente invasivas. Videocirurgia. Ambientes de apoio a construção de conhecimento e aprendizagem . Padrão SCORM ${ }^{T M}$.

Abstract: Today, there is a kind of surgery very popular called minimal invasive surgery (videosurgery), in which the surgeons need to learn and acquire abilities to execute radically different procedures of those existing ones in the traditional surgeries. So, it is necessary to find news alternatives to help the learning of these new tasks. Virtual Reality is one of these possibilities. Besides, it's necessary to make available these environment to community. Learning Objects, Virtual Reality based and SCORM ${ }^{T M}$ compliant, are a very interesting option to make possible to distribute high qualified environments to aid videosurgey technics learning.

Keywords: Learning Objects. Knowledgment. Digital Artifacts. Virtual Reality. Medical education. Minimal invasive surgery. Videosurgery. Learning suport environment. SCORM ${ }^{T M}$ compliant.

\footnotetext{
* Professor, Doutor em Informática na Educação, Centro Universitário Ritter dos Reis, e-mail: franca@ ritterdosreis.br
} 


\section{$1 \quad$ Introdução}

A prática do uso intensivo de tecnologia para criar ambientes que proporcionem melhores condições para a aquisição e construção do conhecimento está sofrendo uma incrível expansão. $\mathrm{O}$ uso da tecnologia está possibilitando às instituições de ensino realizar experiências para além da noção popular de "sala de aula" e, sem dúvida nenhuma, os benefícios obtidos até agora com esta prática são inegáveis. Os diversos problemas, que no entanto emergiram, não apontam para um arrefecimento nesta expansão. Com certeza, os esforços de pesquisa que se fazem necessários certamente encontrarão soluções para eles.

Entre as novas tecnologias em expansão, está a Realidade Virtual. O uso dessa emergente e promissora tecnologia, que faz parte dos chamados Sistemas de Realidade Virtual (os SRVs), vem se difundindo de forma muito rápida na educação.

O uso da tecnologia como um agente de mudança na praxis educacional trouxe inúmeros desafios, entre eles está a implementação de técnicas que possibilitem formas de projetar, desenvolver e distribuir material educacional para todos os que quiserem aprender. Uma tecnologia voltada para a educação, chamada Objetos Educacionais (Objetos de Aprendizagem) tem se mostrado robusta, prática, econômica e forte candidata a liderar as alternativas de escolha para esta área, devido ao seu potencial de reusabilidade, generalidade, adaptabilidade e escalabilidade. Objetos de aprendizagem são elementos de um novo tipo de instrução baseada no paradigma da orientação a objetos.

\section{Sobre as videocirurgias}

A medicina tem alcançado, até os dias de hoje, significativos avanços. No entanto, o uso de artefatos no apoio à educação e prática médica não tem evoluído com a mesma velocidade. No aprendizado de técnicas cirúrgicas, apenas para citar um exemplo, ainda se continuam usando ambientes e instrumentos que muito pouco mudaram nestes últimos anos.

As cirurgias se dividem em dois grandes grupos:

- Cirurgias "a céu aberto" e

- Cirurgias minimamente invasivas.

As Cirurgias "a céu aberto" englobam todas as cirurgias tradicionais. Nas Cirurgias Minimamente Invasivas (CMIs), também chamadas de Videocirurgias, instrumentos cirúrgicos longos, finos e rígidos, e uma microcâmera de vídeo endoscópica são introduzidos no paciente. A partir das imagens produzidas pela microcâmera, e reproduzidas em um monitor de televisão, o cirurgião executa os procedimentos cirúrgicos (figura 1). Neste tipo de cirurgia, os procedimentos são altamente complexos. Para o seu aprendizado, o exercício é fundamental.

\section{Sobre a Realidade Virtual na educação médica}

A Realidade Virtual está proporcionando, sem sombra de dúvida, algumas mudanças no processo educacional. Como um ambiente de apoio a construção de 
conhecimento e aprendizagem, os mundos virtuais, onde o sujeito pode se movimentar, ouvir, ver e manipular objetos, como no mundo real, certamente representam interessantes oportunidades à disposição dos educadores. A introdução da Realidade Virtual na educação modificará significativamente o papel desses educadores; exigirse-á deles novas competências. É necessário, no entanto, que eles se sintam motivados a recorrer ao uso desta nova tecnologia e que pesquisas continuem a demonstrar ser esta, realmente, mais uma alternativa para o sujeito da aprendizagem.

A Realidade Virtual, como artefato de apoio ao processo de habilitação de profissionais em cirúrgias é, atualmente, bastante utilizada, porém de forma experimental. Existem alguns poucos protótipos comerciais disponíveis. Através de instrumentos como o PHANTOM, que simula artefatos utilizados nas videocirurgias, é possível criar ambientes de aprendizado nos quais o procedimento real é simulado. Os instrumentos destes ambientes podem capturar as ações (força e movimento) do aprendiz, apresentando imagens em um monitor de vídeo.

\section{Sobre os objetos educacionais}

O paradigma da orientação a objeto, utilizado na programação de computadores serviu de base para os Objetos Educacionais (Objetos de Aprendizagem), componentes de apoio à construção do conhecimento e aprendizagem humana e que podem ser usados em múltiplos contextos. Esta é a ideia fundamental por trás dos objetos de aprendizagem. Professores e projetistas de conteúdo podem construir pequenos componentes que podem ser utilizados inúmeras vezes e em diversos contextos de aprendizagem. Objetos de aprendizagem são distribuídos através da Internet e intranets, podendo ser disponibilizados simultaneamente para um considerável número de pessoas. Além disto, professores e projetistas de conteúdo podem colaborar com novas versões, beneficiando-se de forma cooperativa (LTSC, 2003).

\section{Sobre o padrão SCORM}

Em 1977 o Departamento de Defesa americano estabeleceu o Advanced Distributed Learning Initiative com o objetivo de desenvolver um estratégia para o uso de tecnologia de informação e aprendizagem para modernizar a educação e treinamento, bem como promover uma estreita cooperação entre governo, academia e a industria para estabelecer uma estandartização de componentes que pudessem ser usados nos ambientes virtuais de aprendizado. Desta iniciativa resultou a definição de uma série de especificações e requerimentos de alto nível para objetos de aprendizagem, que promovesse conteúdo reusável, acessibilidade, durabilidade e interoperabilidade. Ao conjunto destas especificações e requerimentos chamou-se SCORM $^{\mathrm{TM}}$ - Sharable Content Object Reference Model.

O Sharable Content Object Reference Model $\left(\right.$ SCORM $\left.^{\mathrm{TM}}\right)$ define um modelo de agregação de conteúdo e um ambiente de execução, baseado na $W E B$, para objetos de aprendizagem. É um modelo que referencia um conjunto interrelacionado de especificações técnicas e um guia para atender aos requerimentos de alto nível para conteúdo de aprendizado, baseados na WEB, exigidos pelo Departamento de Defesa do governo americano. 
O ponto chave no padrão SCORM ${ }^{\mathrm{TM}}$ é o SCO (SHARABLE CONTENT OBJECT). Um SCO pode ser definido como uma peça instrucional independente e é a menor unidade lógica de instrução que pode ser distribuida e mapeada via um LMS (Learning Management System). Um SCO poderá conter objetivos de aprendizagem, coleção de objetivos de aprendizagem, testes, cenários, simulações, etc. Um SCO é uma coleção de ASSETs, que são representações eletrônica de mídia, texto, imagens, sons, páginas WEB, simulações e outras peças de dados que podem ser distribuídos para um cliente WEB (ADVANCED, 2003).

\section{Sobre a integração de objetos educacionais e Realidade Virtual}

Uma das limitações existentes atualmente na produção de objetos de aprendizagem baseados na Realidade Virtual e compatíveis com o padrão SCORM reside na necessidade de se ter uma intensa interação entre o usuário e o sistema, característica fundamental nos sistemas de Realidade Virtual. Os objetos educacionais "scormizados" precisam de "browser" para executá-los e/ou visualizá-los. Entretanto, os browses, hoje, não interagem com a maioria dos dispositivos necessários à Realidade Virtual (luvas, óculos, capacetes, dispositivos tipo PHANTOM,etc...). No momento, esta limitação está sendo contornada utilizando-se a Virtual Reality Modeling Language (VRML), já que a utilização da VRML supre, em parte, aquelas limitações e é suportada por boa parte dos "browsers" atuais. Pesquisas estão sendo realizadas visando aumentar o grau de interação entre estes "browsers" e os dispositivos exigidos pela Realidade Virtual.

\section{$7 \quad$ Sobre os objetos educacionais e a aprendizagem de videocirurgias}

A aprendizagem de técnicas videocirúrgicas se dá basicamente através da participação de palestras, leitura de textos, visualização de vídeos e figuras e, principalmente, através do exercício. Estes exercícos são fundamentalmente os seguintes:

- manejo dos artefatos cirúrgicos;

- trato do efeito fulcrum (efeito responsável pelo movimento contrário das mãos do cirurgião);

- aplicação de clips;

- dissecação de veias;

- sutura;

- incisões;

- coagulação.

Independente do tipo de videocirurgia, alguns procedimentos são comuns. Outros são específicos. Sendo assim, é possível utilizarmos o conceito de objetos educacionais, e usufruir de umas das principais caracaterísticas destes objetos: reusabilidade. Projetando-se objetos educacionais independentes de contexto, é possível reutilizá-lo em diversas situações. Desta forma, se desenvolvermos objetos educacionais "scormizados" baseados em Realidade Virtual, já que, ao simular o real, a Realidade Virtual, pode proporcionar situações equivalentes ao real, condição necessária para o aprendizado (FERREIRA, 2003), será então possível produzir e disponibilizar, para 
uma comunidade bastante ampla, ambientes de apoio a construção de conhecimento e aprendizagem de técnicas videocirúrgicas altamente qualificados.

As figuras 2, 3 e 4, mostram alternativas para a estruturação de um ambiente com esta finalidade (LEARNING, 2003). Os ASSETs seriam compostos, além de vídeos, textos e figuras, também de simulações dos procedimentos a serem aprendidos e/ou exercitados.

\section{Conclusões finais e trabalhos futuros}

O uso de Realidade Virtual em ambientes de apoio a construção de conhecimento e aprendizagem de técnicas videocirúrgicas tem se mostrado efetivo, desde que simule situações equivalentes ao real (FERREIRA, 2003). A possibilidade de produzir-se estas simulações com caracteristicas de objetos educacionais e de acordo com o padrão SCORM ${ }^{\mathrm{TM}}$, com certeza vai potencializar ainda mais estes ambientes de apoio a construção de conhecimento e aprendizagem. Entretanto, como vimos, existe ainda a necessidade de dotarmos os browsers atuais com funcionalidades que melhorem a interação com os dispositivos de Realidade Virtual. O autor está conduzindo pesquisa neste sentido e espera ter, rapidamente, resultados significativos neste sentido.

\section{Referências bibliográficas}

ADVANCED, D. L. The SCORM Overview. Advanced Distributed Learning Initiative. Disponível em: http://www.adlnet.org/ , acessado em 05/09/2003.

ADVANCED, D. L. The SCORM Run-Time Environment. Advanced Distributed Learning Initiative. Disponível em: http://www.adlnet.org/ , acessado em 05/09/2003.

FERREIRA, Luis de França. Condições para o Fazer e Compreender na Realidade Virtual. Tese de doutorado. Pós-Graduação em Informática na Educação. UFRGS. PortoAlegre, 2003.

LEARNING Systems Architeture Lab. SCORM Best Practices Guide for Content Developers. Carnigie Mellon University. Disponível em: http://www.lsal.cmu.edu/lsal/expertise/projects/developersguide/ acessado em 05/09/2003.

LTSC. Learning technology standards committee website [On-line]. Disponível em: http://ltsc.ieee.org/, acessado em 04/09/2003. 